\title{
Encouraging Active Learning in Higher Education: A Self-Determination Theory Perspective
}

\author{
Lucas M. Jeno \\ bioCEED - Centre of Excellence in Biology Education \\ Department of Biology, University of Bergen, Norway
}

\begin{abstract}
Based on the work of Self-Determination Theory, this article suggests how to implement SelfDetermination Theory based principle in a learnercentered perspective. Higher education has traditionally rested on learning methods that render passive students. Societal changes require selfregulatory skills and an active motivational set. However, lack of theoretical, empirical and practical driven theory in implementation of learner-centered education has lead to a philosophical debate. It is argued for a holistic model for implementing principles derived from Self-Determination Theory (SDT) in a learnercentered paradigm. SDT makes specific prediction for nurturing vs. neglecting learning environments, and thus highly appropriate framework. An important differentiation between types of motivations that differs in relative autonomy, and social climates that may be perceived as amotivating, controlling, and informational is necessary for understanding learning and educational practices. Finally, practical recommendations for teachers in higher education to put into practice. It is argued for a system in which all levels of education supports motivation to support student motivation. Both the institutionallevel and teacher culture must have a learnercentered perspective, further, pre-during-post class preparations are important for high quality learning.
\end{abstract}

\section{Introduction}

Transformation from industry-based society to knowledge-based society demands the need for skills such as critical thinking, explorative abilities, creative thinking, and transferable skills. Skills such as automatic and rudimentary knowledge may be unproductive and undesirable in the future. A child born this year enters the labor force in 26 years if he graduates with a master degree, as many students do, at least in Scandinavia. Do they possess the necessary skills to meet new challenges and create sustainable solutions? How are we preparing the students?
Students have a natural inclination to learn and actively integrate knowledge into a coherent knowledge base. Specifically in higher education, students may choose a subject or major based on interest or curiosity, personal goals, or ambitions. Hence, the possibilities for learning and teaching students, based around their own interest are present. However, some students may find themselves at their university or college learning passively without passion and meaning. They lack agency and authenticity, and are often alienated or helpless learners. What motivational and psychological functions prompt such processes? How could teachers inspire students to reach their highest potential for human functioning?

Learning may be defined differently and implies several aspects. The spectrum of learning perspectives ranges from socially constructed to biological embedded. For example, Wren and Wren [1] asserts that learning is not dependent on teaching, while the opposite is true. Furthermore, education may be historically, cultural, and context dependent. This shows the complexity of learning in an educational context. One way to contrast learning is on active and passive learning [2]. While the latter refers to passively receiving information, the former require the learner to interact with the material, either analyzing, comparing, making inferences, or evaluating critically. Though the active/passive dichotomy can be paralleled with learner-centered and teacher-centered education, the former may be a consequence of learnercentered vs. teacher-centered education. Learnercentered education, as opposed to teacher-centered education, has previously characterized as a perspective on learning where the main focus is on the learner and learning process not on the teacher, where students take responsibility, and finally where formative assessment is implemented for learning and not as means for teaching for tests [35].

Barr and Tagg [3] argue for a paradigm shift, from instruction to learning. The authors list a number of measures to be taken in order to shift from the instruction paradigm to learning paradigm (e.g. improve instruction vs. improve learning). Similarly, Gibbs [4] highlights the importance of 
shifting the main focus from teaching to learning; from the classroom to learning environment; from the individual teachers to focus on leadership and teams, amongst other. Most importantly, there should be a shift from an atheoretical focus to conceptualized and theorized focus [4]. Albeit, learner-centered education has been investigated within the constructivist paradigm of learning, a lack of theoretical framework has been missing for both testing and implementation.

The motivational theory Self-Determination Theory (SDT) has through decades of investigations endeavored such issues [6, 7]. Research guided by SDT has addressed which factors affects motivation, and which effect this has on learning (social climate $\rightarrow$ motivation $\rightarrow$ consequences). Can active learner-centered education be explained from a SDT point of view? And how can we implement learner-centered education in a Self-Determination Theory perspective?

In higher education, a traditional large-group lecture is often the chosen learning method when introducing a subject or teaching students in higher education [8]. Contradictory to research, the argument rest on assumptions of effective transmitting knowledge, lower economical costs, and previous tradition and expectations from both teachers and students [8]. A traditional lecture depends on both attention and interest from the students. Why should students in higher education be active? Do students' learning benefit from a learner-centered education?

This article will address these abovementioned questions in turn. Firstly, Self-Determination Theory is presented as a theoretical framework in order to systemize learner-centered education as a learning perspective. Secondly, a string of argument will be presented as to why higher education should encourage active learner-centered education. Lastly, practical pedagogical implications will be presented. This last point has been lacking in many discussions on implementing learner-centered education.

\section{Self-Determination Theory}

Self-Determination (SDT) is an organismic dialectic theory on motivation that assumes satisfaction of three basic universal psychological needs for psychological well-being [6, 7]. Specifically, autonomy refers to feeling volition, choice in one's behavior while having an internal perceived locus of causality. Competence refers to feeling efficacious in the interaction with the environment. Lastly, relatedness is the feeling of being cared for, belonging, and cared for by others. Satisfaction of the basic needs for autonomy, competence, and relatedness are necessary for intrinsic motivation and growth. Thwarting of the needs is associated to hinder growth and foster psychological ill-being. A student that is performing a learning activity out of choice and interest, fully endorsed, receiving effectance relevant feedback, from a supportive teacher is theorized to satisfy his/her psychological needs.

In higher education however, not all learning activities, subjects or classes may be intrinsically motivated. According to SDT, the degree of selfdetermined motivation is important for the quality of that learning activity. SDT distinguishes between three classes of motivation in terms of how internalized the behavior is, and how selfdetermined it is (Figure 1) [9]. Controlled motivation is the least self-determined class of motivation, and consists of external regulation and introjected regulation. Controlled motivation refers to behaviors that are performed in order to obtain a reward or avoid a punishment (external regulation), or performed to avoid guilt or attain self-worth (introjected regulation. Controlled motivation is associated with pressure, coercion, and tension and is assumed to be negative related to well-being, performance, and satisfaction.

Autonomous motivation, on the contrary, is a self-determined type of motivation and is associated with high quality functioning, performance, and well-being. Autonomous motivated students endorse and value the behavior or goal because is personally important (identified regulation), or has full internalized it into congruence with the self and value system (integrated regulation). Intrinsic motivation is the prototype of self-determined motivation and refers to doing a behavior out of the inherent interest and fun of the activity. It is important to note that the internalization process is not stepwise or age-dependent. A student's motivation may fluctuate from intrinsic motivation to external regulation without going through the integrated, identified, and introjected. Similarly, initial disinterest in a subject may become highly autonomously motivated under the right conditions.

Several investigations have found support for SDT's basic tenets in a pedagogical context. In a study by Jeno and Diseth [10] the authors found in a study among upper-secondary support for a SDTbased path-analysis. Specifically, students that where autonomous motivated for learning and perceived themselves as competent to learn, had a positive relation with perceived school performance, conversely, controlled motivated students had a negative relation to perceived school performance. These relations have been found both in correlational and experimental studies, across all school ages $[6,11]$.

\subsection{Social climate}

Learning in higher education is not solely determined by students' personal motivation, but 
also to the extent that the teachers able to support or hinder learning and motivation. In some instances, an unmotivated student may be inspired and encouraged by a teacher or lesson. Similarly, an uninspiring teacher or lesson may discourage a student toward learning.

According to SDT's dialectical framework, motivation is influenced by the students' social context and climate. Hence, a classroom situation or learning activity may be perceived differently by the students and thus have different functional significance, informational, controlling or amotivating, respectively $[9,12]$. An informational aspect conveys autonomy support, that is, it provides the student with feeling of autonomy, enhances competence, and encourages selfdetermined motivation. A functional significance is controlling when the event pressures the students and controls toward a specific learning activity. Lastly, an amotivational functional significance conveys incompetence and helplessness with lack of autonomy and relatedness. In examples, an informational teacher would facilitate the learning process by providing the students with choice, provide the students with positive effectance relevant feedback that is directed towards their personal goals, while trying to understand students' internal frame of reference.

Both experimental studies and correlational studies have found support for supporting autonomy in an informational aspect, while the opposite is true for the controlling aspect. In a study with a college sample, students who perceived their instructor as informative and autonomy supportive not only became more adjusted in that course and performed at a higher level, they became more autonomous motivated across the course [13]. Similar results have been found for secondary students, upper secondary students, and college students [10, 14, 15].

In sum then, students benefits when in an informative and autonomy supportive environment. Teachers that supports students' psychological needs for autonomy, competence, and relatedness, leads the students to become autonomously motivated, and finally, perform better at school and have higher well-being.

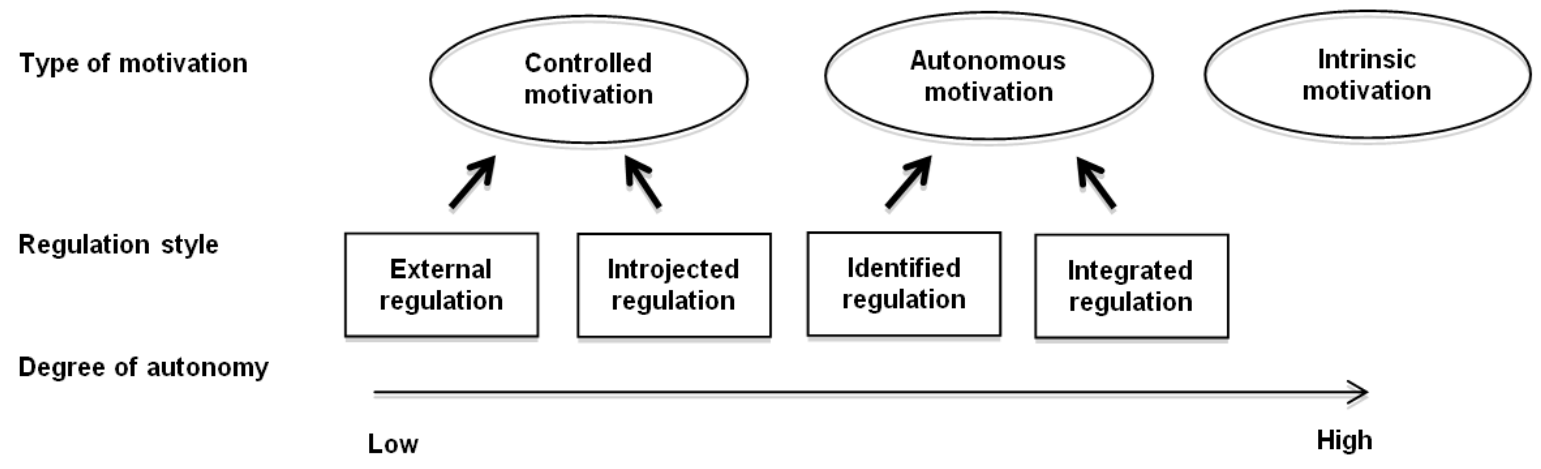

Figure 1. Internalization process

\section{Discussion}

The main goal of the current discussion is to highlight the importance of having a clear theoretical stand when implementing pedagogical practices. Specifically, when implementing learnercentered education, previous suggesting has lacked a theoretical empirical drivel framework. Learning in higher education encompasses an array of processes. Different scholars have argued for a shift from passive instruction teaching, to active learner education. However, a lack of theoretical positioning implies an un-coherent methodology on how to facilitate such active learning. Based on the abovementioned theoretical framework, SDT offers clear theoretical and empirical assumptions for learning. SDT's organismic perspective assumes that students are active and intrinsically curious and explorative individuals. Students that are intrinsically motivated for learning in a course or subject are guided by their intrinsic motivations.

A second aim of the present article is why higher education should encourage active learners. As mentioned at the beginning, it might a myth that traditional methods are more effective and economical effective. For example, Benware and Deci [16] found in an experimental study that students who where told to learn a material to teach other student (active learning) did not differ significantly in rote learning compared to students who learned to perform at a high standard (passive learning), however the active-condition performed 
better than the passive-condition in conceptual learning and rated higher intrinsic motivation. Prince [17] performed a study investigating the efficiency of active learning. The results shows that the introduction of active learning in class not only leads to retaining and understanding knowledge, it increased engagement for learning. In a similar vein, collaborative learning, which could be characterized as active learning, show strong effect sizes (0.44-0.70) compared to learning individually [17]. According to Prince [17], active learning may be as simple as comparing notes during class with peers. Mostrom and Blumberg [5] argues that, though learner-centered education may increase achievement compared to teacher-centered education, it is important to distinguish between higher performance mean due to inflation and due to gaining knowledge. The latter is a desirable for learning and education.

Active learning may be beneficial because they are engaged with the material and more involved with the learning process. Autonomous and intrinsic motivated students are guided by interest, seeking optimal challenges, enjoyment, and importantly, choice. When the behavior is regulated by the Self, the student has internalized the behavior and made it a part of their own value system and regulation from teachers is not needed. This is an important point for future student that are required to think creatively, critically, and outsidethe-box. SDT's dialectical perspective argues that students' motivation is influenced by their perception of motivators. As mentioned earlier, according to SDT and environment could either be informative, controlling, or amotivating. In which case, SDT propose different outcomes in terms of learning and behavioral consequences. According to Ryan and Deci [9], teachers support may prompt and sustain the internalization process. This is because teacher can relate to students at a personal and affectionate level.

\section{Practical implications for higher education}

The last goal of the article is to offer practical implications for implementing active learnercentered education. Based on the theoretical framework and research presented, this section will provide practical implication for educators in higher education and how to facilitate learning and support motivation at different levels (Figure 2).

Institutional-level: Ryan and Weinstein [12] asserts that different ambitions of outcomes in regard to teaching and learning may have different motivational consequences. In example, a University may receive more or less funding depending on test-scores and examination rates. Research based on SDT find that such "highstakes" measures are ineffective in terms of higher academic achievements and psychological wellbeing $[12,18]$. Several explanations are put forth. Firstly, rewarding or punishing competence pressures both students and teachers, and thus thwarts the need for autonomy and competence. Secondly, never reaching standard, while continually being punished may lead to a amotivation functional significant aspect and in the long run higher dropout rates.

It is important that leaders at the institutionallevel have an informative aspect on teaching and education. According to Pelletier and Sharp [19], teachers autonomous motivation for teaching may be influenced by leaders relative provision of autonomy support or neglect. Both time constraints and curriculum constrains may be perceived as controlling and thus not supporting of autonomy. The may in turn inhibit teachers autonomy and finally autonomous motivation for teaching. Pelletier and Sharp [19] asserts that autonomous motivation for teaching is associated with students' own autonomous motivation for learning.

Teacher-culture: What type of educational perspective do you as a teacher have? What are your views on cognition, motivation, developmental and social factors, and individual differences [20]? Is your view on intelligence, motivation, and learning as fixed, mechanic and could direct by external contingencies? Or is your perspective on intelligence, motivation, and learning, as a natural and an inherent propensity towards growth and integrating that needs support and nutriment for healthy functioning. If your perspective is on the latter, an organismic or liberal perspective on education, you are likely to view learning as an active process that may be fostered by a learner-centered education. Another important point for teacher culture is on fellow teacher colleagues. Supporting each other's needs for autonomy, competence, and relatedness is important for a structural organizational change along with leaders support.

Pre-lesson: What are your goals for this particular lesson is it part of a long term goal or philosophy of education? Teachers may identify students' personal interest and base the lesson around their interest in order to support their intrinsic motivation. For controlled motivated students, teachers are encouraged to reflect on how the lesson could be more valuable for the students. How those this subject relate to their education and aims for their degree and education?

During the lesson: There are several measures that can be taken to increase autonomous motivation [6, 9]. For example, teachers can provide students with moderately challenging tasks. When facing uninteresting tasks or over/under challenging activities, teacher are recommended to acknowledge students negative affects and provide them with an informative rational as to why the task 
or activity is important. Students may then internalize and integrate the behavior in their value system and move from controlled to autonomous motivation.

Post-lesson: What did you do during your teaching lesson? Can you imagine how your students perceived your lesson? Reflection afterwards a lesson is highly important in order to evaluate and critically assess which parts of the lesson went well and which parts could be improved. A possible solution to receive feedback is to use electronic student polls and ask them to assess on a scale if they learned during the lesson. Furthermore, you could ask control question from reading assignments and from the lesson in order to assess if the students have understood the lecture.

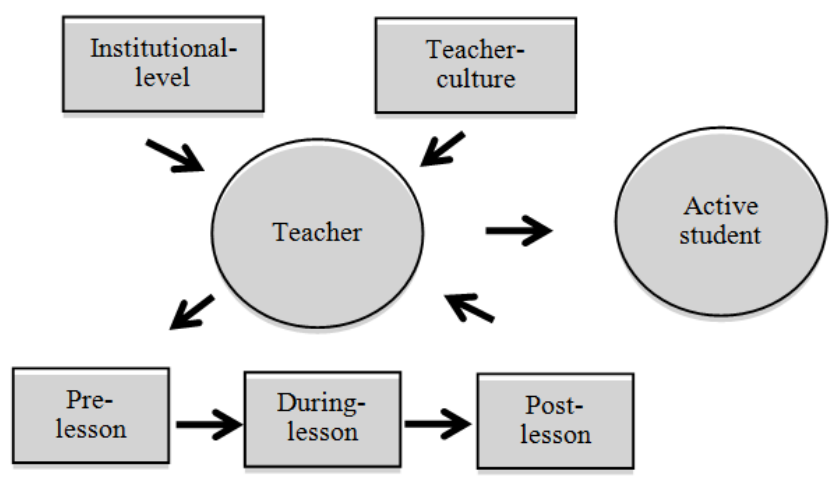

Figure 2. Practical framework for active students

\section{Conclusion}

In sum then, learner-centered higher education is recommended for active students. Active students, as opposed to passive students, are more engaged and interested in their learning. As mentioned above, a learner-centered education is an important step towards increasing active student. This change should be driven by a holisticdialectical, empirical driven theory. SelfDetermination Theory offers an organismic and dialectical perspective on human motivation and personality with testable hypothesis that makes clear prediction of antecedents of motivation and learning, and outcomes and consequences of different types of motivation. Teachers are recommended to support autonomy, competence and relatedness for students to develop an autonomous motivation for learning. However, all aspects of learning and education must be considered for effective change.

\section{References}

[1] Wren, C. and Wren, T. (2003) 'The Capacity to Learn, in A Companion to the Philosophy of Education', in R. Curren, Editor., Blackwell Publishing Ltd: Oxford. p. 246-259.

[2] Ferguson, K.J., (2010) 'Facilitating Student Learning, in An Introduction to Medical Learning', in W.B. Jeffries and K.N. Hugget, Editors., Springer: New York. p. 1-10.

[3] Barr, R.B. and Tagg, J. (1995) From teaching to learning - A new paradigm for undergraduate education'. Change, 27(6), p. 12-26.
[4] Gibbs, G., (2009) 'Developing students as learners varied phenomena, varied contexts and a developmental trajectory

for the whole endeavour'. Journal of Learning Development in Higher Education (1), p. 1-12.

[5] Mostrom, A.M. and Blumberg, P. (2012) 'Does Learning-Centered Teaching Promote Grade Improvement?' Innovative Higher Education, 37(5), p. 397-405.

[6] Ryan, R.M. and Deci, E.L. (2000) Intrinsic and extrinsic motivations: Classic definitions and new directions'. Contemporary Educational Psychology, 25(1), p. 54-67.

[7] Deci, E.L. and Ryan, R.M. (1985) 'Intrinsic Motivation and Self-Determination in Human Behavior'. Plenum Press, New York.

[8] Jeffries, W.B. (2010) 'Teaching Large Groups', in An Introduction to Medical Teaching, W.B. Jeffries and K.N. Hugget, Editors., Springer: New York.

[9] Ryan, R.M. and Deci, E.L. (2002) 'An Overview of Self-Determination Theory: An Organismic-Dialectical Perspective', in Handbook of Self-Determination Research, E.L. Deci and Ryan, R.M. Editors., The University of Rochester Press: New York. p. 3-36.

[10] Jeno, L.M. and Diseth, A. (2014) 'A selfdetermination theory perspective on autonomy support, autonomous self-regulation, and perceived school performance'. Reflecting Education, 2014. 9(1), p. 1-20.

[11] Deci, E.L., Koestner, R. and Ryan, R.M. (2001) Extrinsic rewards and intrinsic motivation in education: Reconsidered once again'. Review of Educational Research. 71(1), p. 1-27. 
[12] Ryan, R.M. and Weinstein, N. (2009) 'Undermining quality teaching and learning. A self-determination theory perspective on high-stake testing'. Theory and Research in Education. 7(2), p. 224-233.

[13] Black, A.E. and Deci, E.L. (2000) 'The Effects of Instructors' Autonomy Support and Students' Autonomous Motivation on Learning Organic Chemistry: A Self-Determination Theory Perspective'. Science Education. 84, p. 740-756.

[14] Ntoumanis, N., (2005) 'A Prospective Study of Participation in Optional School Physical Education Using a Self-Determination Theory Framework'. Journal of Educational Psychology. 97(3), p. 444-453.

[15] Próspero, M.s. and Vohra-Gupta, S. (2007) 'First Generation College Students: Motivation, Integration, and Academic Achievement'. Community College Journal of Research and Practice. 31(12), p. 963-975.

[16] Benware, C.A. and Deci, E.L. (1984) 'Quality of Learning With an Active Vesus Passive Motivational Set'. American Educational Research Journal. 21(4), p. 755-765.

[17] Prince, M., (2004) 'Does Active Learning Work?' A Review of the Research. Journal of Engineering Education. 93(3), p. 223-231.

[18] Ryan, R.M. and Brown, K.W. (2005) Legislating Competence. High-Stake Testing Policies and Their Relations with Psychological Theories and Research', in Handbook of Competence and Motivation, A.J. Elliot and Dweck, C.S. Editors. 2005, The Guilford Press: New York. p. 354-372.

[19] Pelletier, L.G. and Sharp, E.C. (2009) 'Administrative pressures and teachers' interpersonal behaviour in the classroom'. Theory and Research in Education. 7(2), p. 174-183.

[20] Lambert, N.M. and McCombs, B.L. (1998) Introduction: Learner-Centered Schools and Classrooms as a Direction for School Reform', in How students learn: Reforming schools through learner-centered education, N.M. Lambert and McCombs, B.L. Editors, American Psychological Association: Washington, DC. p. 1-22. 